\title{
Dynamic Spectrum Allocation Access Using Cognitive Radio Networks in a Maritime
}

\author{
Dickson E. Onu', Mamilus A. Ahaneku², Michael O. Ezea ${ }^{3}$, Henry O. Osuagwu ${ }^{4}$, Udora N. Nwawelu ${ }^{5}$ \\ 1,2, ${ }^{5}$ Department of Electronic Engineering, University of Nigeria, Nsukka, Enugu State, Nigeria \\ ${ }^{3,4}$ Department of Electrical Engineering, Nigeria Maritime University, Okerenkoko, Delta State, Nigeria
}

\section{Article Info \\ Article history: \\ Received Aug 14, 2020 \\ Revised Aug 16, 2021 \\ Accepted Sep 3, 2021}

\section{Keywords:}

Cognitive radio

Maritime environment

Spectrum allocation

spectrum hole

\begin{abstract}
The maritime environment is unique due to radio wave propagation over water, surface reflection and wave obstruction. In dealing with the challenging maritime environment, a dynamic spectrum allocation access for maritime cognitive radio network through optimization is suggested. Existing works in this area are limited in performance due to the long duration in achieving the probability of false alarm. Matched filtering technique which is known as the optimum method for detection of primary users (PUs) faces the challenge of large power consumption as various receiver's algorithm are needed to be executed for detection. This work provides a platform that enables minimum energy utilization by secondary users (SUs) thereby, enhancing throughput. An algorithm for throughput maximum in spectrum allocation was developed and used based on demand based model. The implementation of the developed model was carried out using Java program and the spectrum analysis using long distance path loss model and adaptive modulation code to estimate the minimum bandwidth of the secondary users. A simulation of cognitive radio mesh network for the testing and validation of the demand based algorithm preference, and also the cognitive radio network traffic was carried out using Cisco packet tracer and results shown on MATLAB. Simulation results indicate that using the demand based algorithm, the throughput rose with time and almost stabilized. This increase and steady throughput indicates effectiveness in the algorithm which shows that the PUs and SUs activities increase as holes' detection effort varies, unlike that of genetic algorithm where the throughput rose gradually, got to a peak value at certain time and then fell which indicates instability in the variation of the throughput. Also, the average throughput of the demand based algorithm is far greater than that of genetic algorithm which shows that demand based algorithm outperforms the genetic by a far greater percentage.
\end{abstract}

Copyright $\odot 2019$ Institute of Advanced Engineering and Science. All rights reserved.

\section{Corresponding Author:}

Mamilus A. Ahaneku,

Department of Electronic Engineering, University of Nigeria, Nsukka, 410001, Enugu State, Nigeria.

Email: mamilus.ahaneku@unn.edu.ng

\section{INTRODUCTION}

Wireless broadband has become a very important part of our everyday life and work, due to the complexity in managing very large amounts of internet data. Attention in this area is needed in maritime network despite the fact that broadband access is very common on land. The difficulty in managing maritime network is the inability to maneuver at sea, constrained bandwidth and the dynamic network topology that warrant specific attention [1]. Unlike the land vehicles, maneuvering at sea is affected by sea surface movement and wave obstructions [2]. This can lead to link breakages as a result of low link stability as well 
as highly variable bandwidth. In spite of the need to achieve performance close to high-speed terrestrial wireless broadband service on land, there is only a perfunctory effort to investigate maritime networks.

The exponentially growing demand for higher rate wireless communications to satisfy the greed for more applications has come to a bottleneck state since the spectral resources are running out; there are almost no more frequency bands to allocate to new systems [3]. The usage of the radio spectrum and the regulation of radio emissions are coordinated by national regulatory bodies like Federal Communication Commission FCC. As part of radio regulation, the radio spectrum is divided into frequency bands, and licenses for the usage of frequency bands are provided to operators, typically for a long time such as one or two decades. With licensed frequency bands, operators often have the exclusive right to use the radio resources of the assigned bands for providing radio services.

From the FCC spectrum inventory, it has been discovered that the range of frequency bands popularly used for commercial wireless communication systems have been almost used up. The implication is that there is a limited available spectrum band for installing new systems with a broad bandwidth. This led to the wrong belief that there is a spectrum crisis. This wrong belief is because we look at the spectrum as a huge band of frequencies that we distribute over different systems using a fixed spectrum assignment policy, but what if we look at the spectrum from the time respective for the same frequency range, We will find that the band utilization in urban areas ranges from less than $1 \%$ to slightly more than $50 \%$ as measured experimentally in [4], and it is even less in suburban and rural areas. This indicates that a huge spectrum opportunity is available for new generation of wireless communication systems if these systems are made to be aware enough of the spectrum in order to recognize and utilize these spectrum opportunities in dimensions other than frequency.

According to [5]-[8], Cognitive radio is an exciting promising technology which has emerged to solve the problem of underutilization of the available spectrum. Since it has been verified that the primary (licensed) users do not use all the spectrum allocated to them all the time, cognitive radio does the work of sensing the empty frequencies in the licensed frequency bands and Secondary Users are allowed to opportunistically make use of the spectrum in as much as it does not result to destructive interference of active Primary Users. This opportunistic and dynamics of communication paradigm results to higher utilization of spectrum and provide Secondary Users with effective source of availability and reliability because they can access any spectrum part without interruption of ongoing PUs transmission and also hop to a different part when required. The assigning of free channels between primary users and secondary users in a specific geographical region while minimizing interference among all users is called dynamic spectrum allocation in cognitive radio. Dynamic allocation of spectrum is directed towards overcoming the problem of underutilized holes or spaces and improvement on inefficiency of fixed sense spectrum holes [9].

Optimization is a fascinating tool to model and handle the problem of spectrum allocation in cognitive radio network. Hence this work proposes the use of optimization method to address the challenges of spectrum allocation for cognitive radio mesh network and maximize theoretical system performance of secondary users subject to limited power dissipation and non-interference of licensed users.

The rest of the paper will be discussed in the following order: Section II talks about the work found from literature. Section III is on methodology and system analysis. It also describes the design concept and principles of cognitive radio wireless network and associated algorithms. Section IV presents the result and discussions. It gives details of the simulated work required for the performance evaluation of the prototype in terms of secondary user's spectrum sensing and validation of the model developed. It also discusses the result and analysis of the work. Conclusion and recommendations are contained in section $\mathrm{V}$.

\section{FUNDAMENTAL CONCEPT OF COGNITIVE RADIO SYSTEMS}

The progressive increase in the number of mobile users in the wireless communication is enough to create a bandwidth crisis due to the limited availability of natural electromagnetic radio spectrum. Cognitive radio is an emerging technology capable of solving the problem of spectrum scarcity in the wireless communication. The solution is provided through efficient spectrum utilization techniques by applying the optimistic sharing techniques. The concept of cognitive radio is seen to be first conceived by Mitola [10]. Figure 2.1 shows the Cognitive Cycle by Mitola.

The operation of a cognitive radio involves four processes: spectrum sensing, spectrum management, spectrum sharing and spectrum mobility [11]. Spectrum sensing is the process of determinig the availability of spectrum in the licensed users. Spectrum management is to predict how long spectrum holes would remain available for use to the unlicensed users. Spectrum sharing is to distribute the available holes fairly among the unlicensed users bearing in mind usage cost. In order to maintain seamless communication requirements during the transition to better spectrum then the spectrum mobility must be properly designed. 


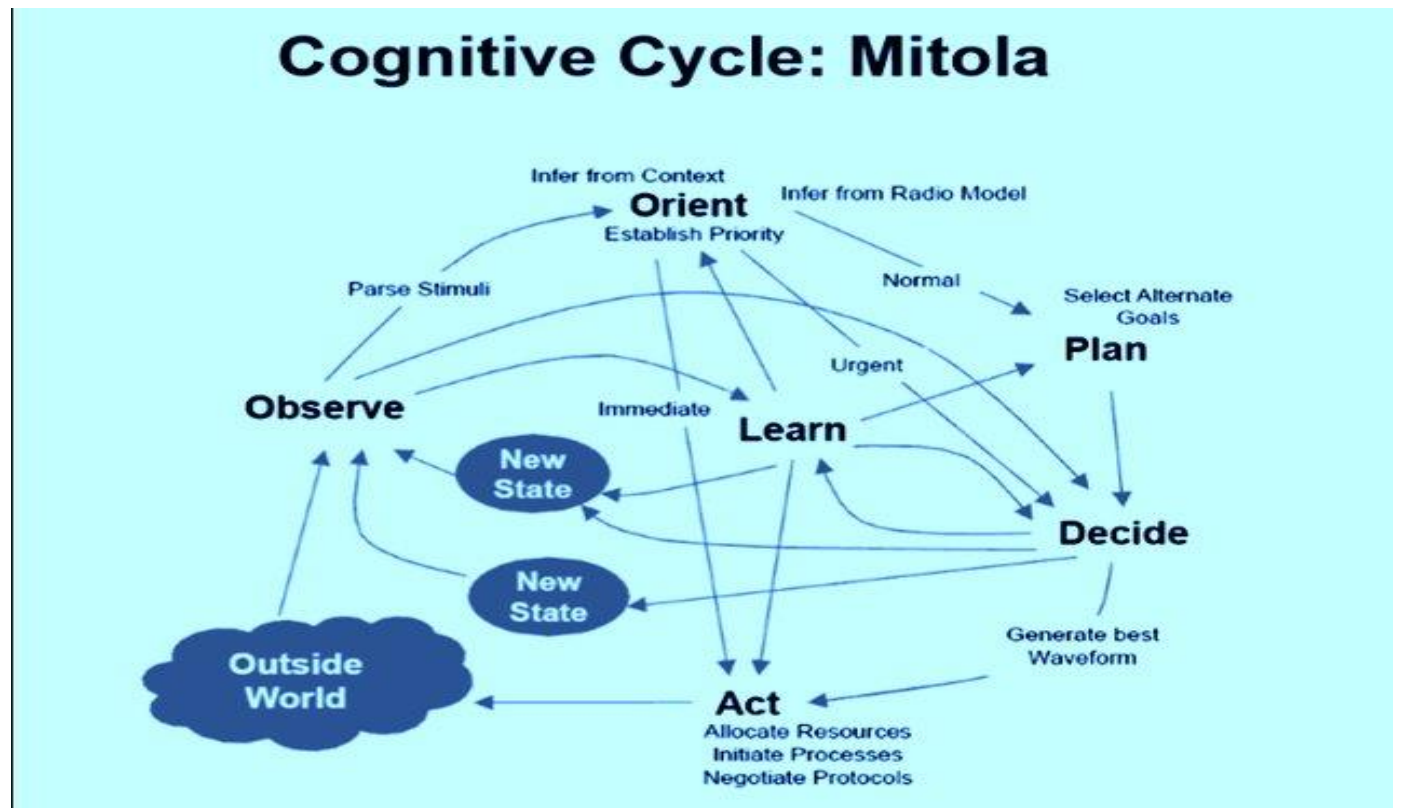

Figure 2.1 cognitive cycle by Mitola [10]

Cognitive radio is characterized by cognitive capability and re-configurability. The cognitive capability enables the finding out of unused radio spectrum at a specific time or location by sensing the information from the environment. Then the selection of the appropriate portion will be carried out for communication without harmful interference to the primary users [12]. Re-configurability helps to dynamically programme the radio according to its environment. To enable it perform its desired functions, the cognitive radio is programmed for transmission and reception on a range of frequencies based on different transmission access technologies supported by its hardware design [13].

\subsection{Channel Modeling in Maritime Cognitive Radio Network}

Current maritime communication systems are based on narrowband UHF and VHF radios for shipto-shore communications near port waters and satellite communication for long-range ship-to-ship and shipto-shore communications [14]. For a normal operation each ship is equipped with cognitive device, which allows them to sense radio environment to access spectrum where the primary users are not active. For instance, ships that are distant from the land, will be very difficult to have access to the Fusion Centre (FC) by the use of either scientific, industrial or medical band on the land. However, the use of satellite link stands as an alternateaccess means to the fusion Centre.

The use of spectrum in cognitive radio network, a ship can adjust its operating parameters in accordance with the secondary user state, geographical location and node density. In [15], the measurement of spectrum in white spaces was carried out for CR networks on the land. Television (TV) and cellular bands are mostly common. On the other hand, further research on the spectrum allocated for maritime communications is necessary, because of the environmental factor. Environmental factors also include the maritime radio atmosphere (sea motion and antenna model). The sea has little or no obstacle, and its surface is flat. These factors are the reasons for huge path loss, due to negative interference between the line of sight (LoS) path and the reflected path [15]. In order to achieve optimal throughput and quality of service (QoS), MCRNs requires the intelligence to adjust their operating parameters to suit the state of the sea, geographic location/region and the range of communication.

\subsection{Cognitive Radio Spectrum Sensing Techniques}

In recent times, different methods of spectrum sensing techniques have been suggested in the literature for the identification of the presence of signal transmission. Some of these approaches are thus: Energy detector based sensing technique, Waveform based sensing technique, Cyclostationary based sensing technique and Radio identification based sensing technique. Due to its low computational and implementation complexities [15], energy detector based sensing technique is the most common method of sensing of spectrum. Some of the challenges with energy detector based sensing, as noted by the authors, including selection of the threshold for detecting primary users, inability to differentiate interference from primary user, noise and poor performance under low signal-to-noise ratio (SNR) value. Waveform-based sensing or coherent sensing technique can only be applied to systems with known signal patterns. Record shows that waveform-based 
sensing outperforms energy detector based sensing both in reliability and coverage time. Also, it was shown that the performance of sensing algorithm increases as the length of the known signal pattern increases [16].

In cyclostationary based sensing technique, instead of power spectrum sensing (PSS), cyclic correlation function is used for the detection of signals present in any given spectrum. The cyclostationary based detection algorithm can differentiate noise from signals of primary users. This is as a result of the fact that noise in wide sense stationary (WSS) with no correlation while modulation signals are cyclostationary with spectral correction due to the redundancy of signal periodicities [17]. For radio identification, feature extraction and classification technologies are used in the context transparent ubiquitous terminal project [18]. The goal is identifying the presence of some known transmission technology and carry out communication via them.

Several of these cognitive radio spectrum sensing approaches proposed in literatures have failed to address the issue of spectrum sensing energy minimization, the constraint in the time sensing duration, and the numbers of SUs per channel. In this work, demand based spectrum allocation algorithm was analyzed to tackled the afformentioned problems.

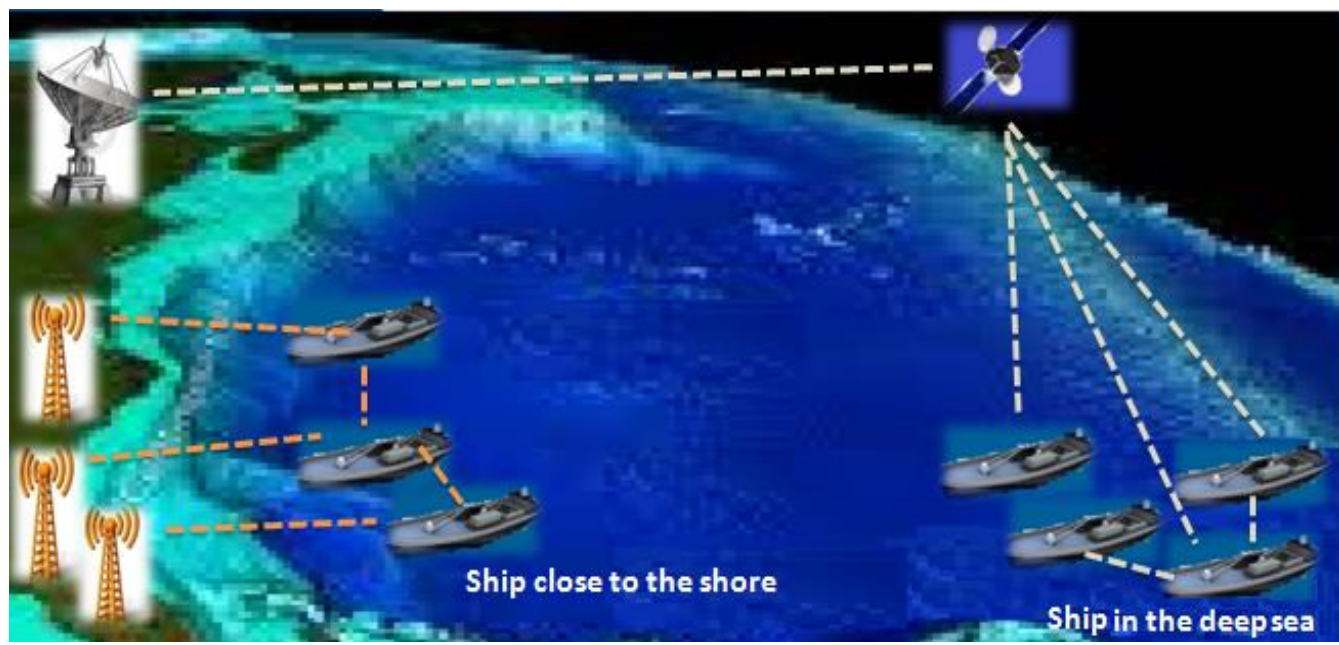

Figure 2.2 Maritime Cognitive Radio Network [15]

\section{METHODS}

In The modeling method adopted here is to exploit a cognitive radio which reduces spectrum sensing energy dissipation in maritime environment. It is a non-coherent detection method that detects the Primary User (PU) signal based on the energy sensed [16]. Due to simplicity and no requirement on prior knowledge of PU signal, energy detection (ED) is the most effective method in cognitive radio sensing. Meanwhile, the model is formulated to tackle the problem of deciding the SUs to be used for the available spectrum. Also, minimal delay improves efficiency [17]. The problem of selecting the spectrum for allocation is tackled using a model which is implemented in java program. Spectrum analysis is tackled using long-distance path loss model and adaptive modulation code (AMC) to estimate the minimum bandwidth of the SUs. Advanced Cisco packet tracer software is also used to build a virtual mesh cognitive radio maritime environment which is used for testing and validation of the proposed spectrum allocation technique. Cognitive mesh radio traffic is simulated within the visual basic software environment. Poisson distributions are used to model the Primary Users (PUs) and Secondary Users (SUs) traffics. The cognitive users are the (PUs and SUs). Transmission arrivals are taken to be Poisson distribution

\subsection{System Component and Model}

Maritime Cognitive Radio Communication (MCRC) from all indications seems to be the best approach for solving the demerit of the maritime broadband wireless communication. The ultra-high frequency band was assumed because it can offer bandwidth beyond $100 \mathrm{MHz}$ opportunistically in maritime network. It also has a communication range at about $10 \mathrm{~km}$. In the MCRCs, the Primary users (PUs) are the analog voice intercom in the VHF maritime band and the legacy system equipment outside the maritime band. The Secondary users (SUs) are the communication equipment device with cognitive radio technology installed on the ship. The ship therefore satisfies the entire requirement for acting as the SUs which include spectrum capability and the reconfiguration of operating parameter such as waveform, spectrum power, power usage, etc. 
Considering the curvature of the earth in the maritime environment, the transmission range of VHF signal is limited, hence, UHF band was assumed to be used. In the region, the SU monitor the working status of the PUs and used the resource of the PUs to exchange data with other SUs. In order to improve the accuracy of detection and solve the competition between SUs, this work built the MCRCs using centralized spectrum allocation scheme. In the scheme, the SU first sends the spectral detection result, before the spectrum lease requesting to Decision-making Center (DC) through an additional signaling channel. Following this, the DC allocates the spectrum using the cognitive algorithm. This work as proposed is based on queuing model. However, according to the distance between the network region and the DC which is built on the land, the system is divided into two typical regions; close to shore and deep sea. In the region close to shore, the SU communicates directly with the DC through the signaling channel. In the deep sea region, there is exchange of data between the SUs and DC through satellite link. Figure 3.1 and Figure 3.2 shows the free body diagram of the illustration. However, an important aspect to be considered of maritime cognitive radio is the protection of the PU.

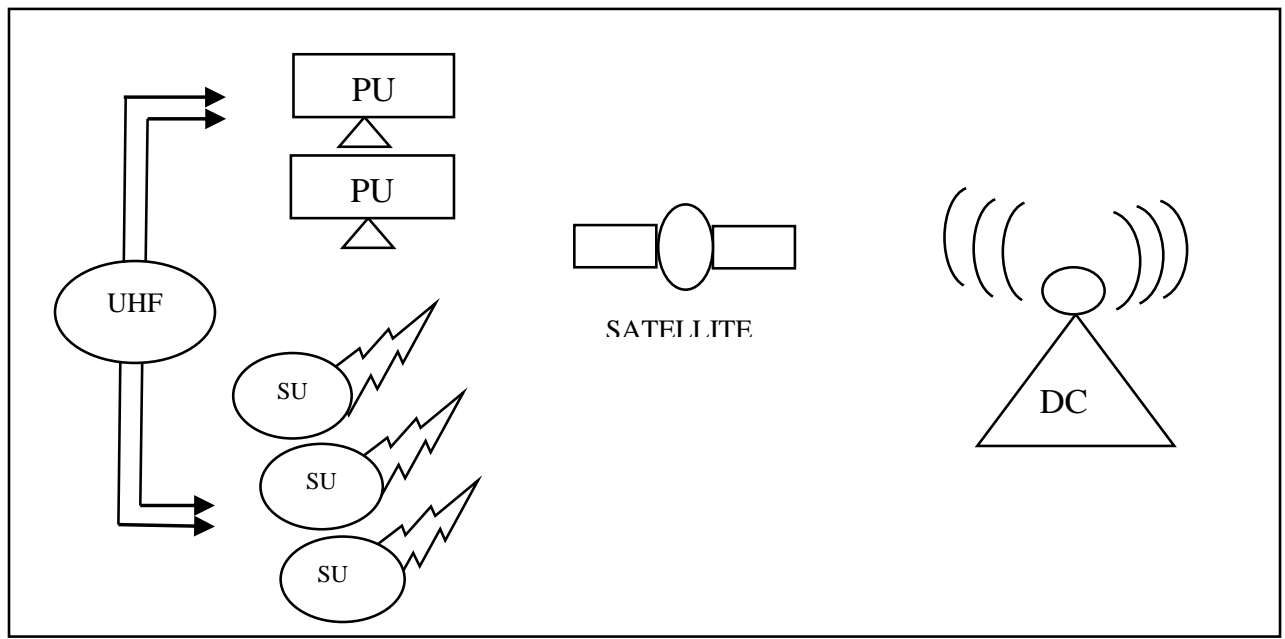

Figure 3.1 Deep Sea Regio

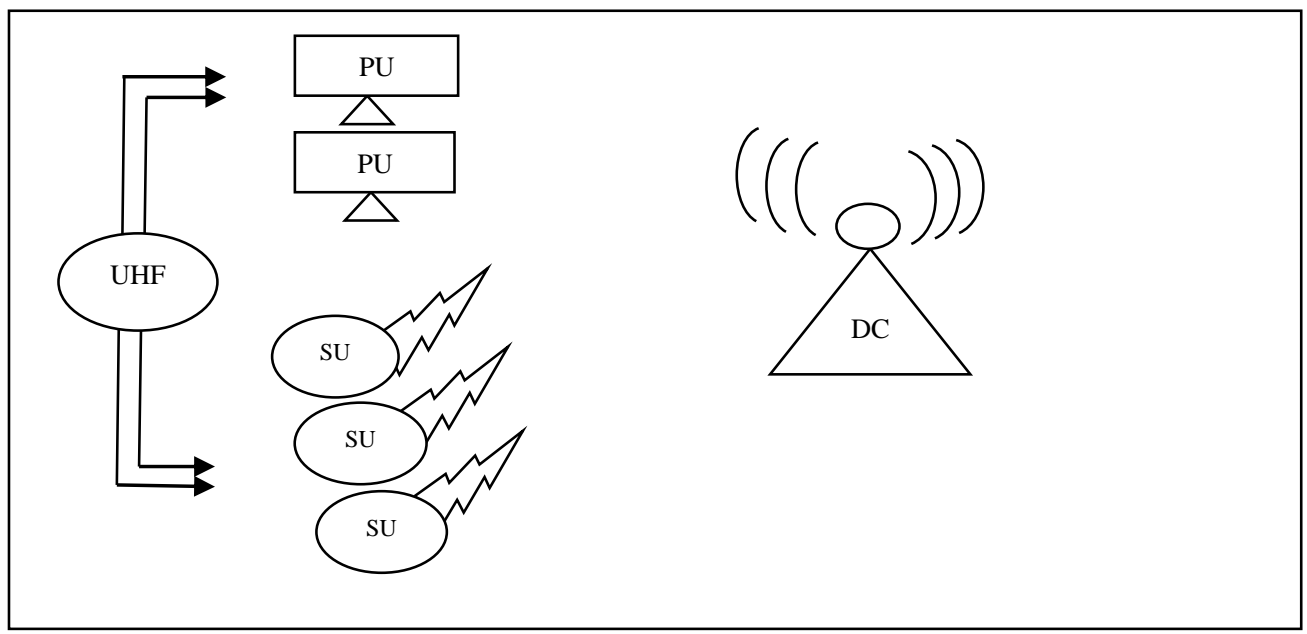

Figure 3.2 Region Close to Shore

\subsubsection{Algorithm Model}

In this work, the goal is to develop an algorithm that is efficient for maritime cognitive radio communication as proposed. Figure 3.1 and Figure 3.2, as shown above, gave pictorial view of the SUs and one PUs system, which include PUs and DC. The PUs have authorized spectrum, which are used to form multiple complex with SUs. Each passes the local energy detects and submits its own detection results to the DC through a unique signaling channel. The DC examines and compares these results to determine the final available free space (hole) and properly allocates the holes to the best available SUs. When the free space or spectrum is allocated, SUs can use adaptive modulating techniques to transmit data in the allocated spectrum. In Figure 3.3, the blue portion represents the spectrum used by PUs that was detected by DC, the red portion represents small fixed frequency band between SUs. The white portion represents the free spectrum 
determined by the DC. Hence, Xi $(i=1,2 \ldots \mathrm{k})$ represent the allocated spectrum size of SUs, which is the strategy of game participants. The DC does not need to obtain the terminal information of the SUs at any moment. When the PU desires to use the spectrum, it will detect the terminal information of SUs, which does not return the spectrum time rate. As a result, the channel resource center can be maximally saved. This work assumed region close to shore scenario(Fig.3.2).

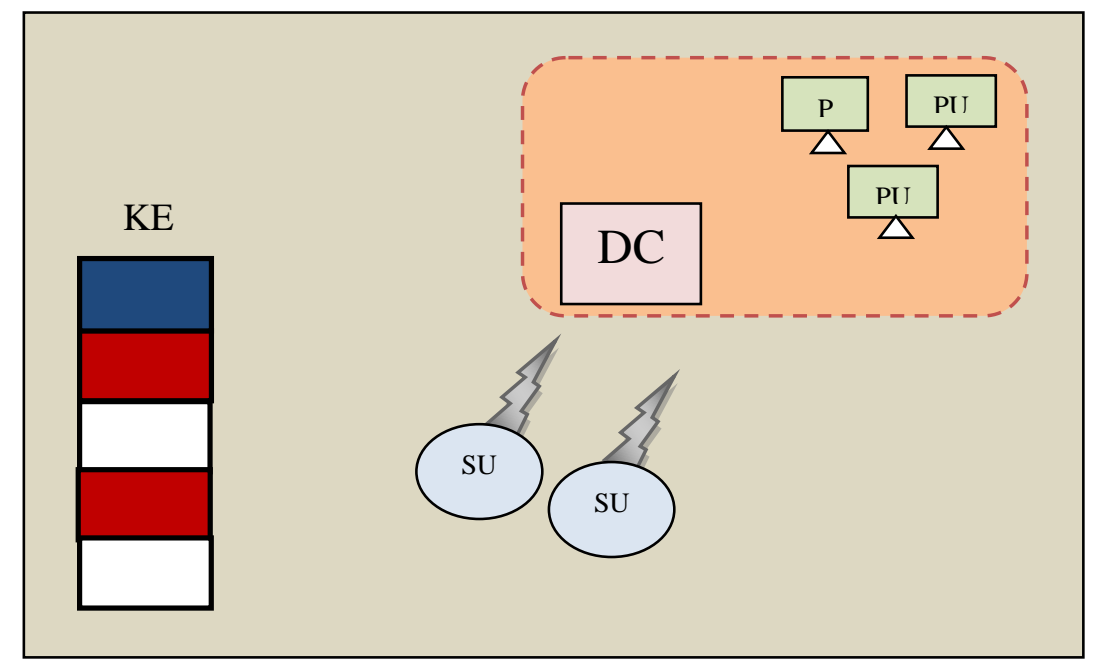

Figure 3.3 Model Allocation Algorithm

\subsection{Demand Based Theory Model}

Let $P^{s}$ and $E_{m, n}^{s}$ be the power consumed during channel sensing and the energy dissipated by SUs for sensing channel respectively. $E_{m n}^{s}=P_{m n}^{s}$. The energy consumption for channel sensing (denoted by $E^{s}$ ) can be written as:

$$
E^{s}=\Sigma^{m} \sum^{n} P_{m n}^{s}
$$

Besides sensing channel, SUs also consume energy transmitting their local result to all the Cognitive Radio Base Station (CBS). It was assumed here that SU transmit its sensing report as a single packet regardless of the number of channel sensed, and the reporting period is long enough that all SUs can find their packets. Let $E_{n}^{r e p}$ denote the energy consumed for reporting the sensing result to CBS, which depends on the location of SUs relative to the CBS. In addition, let $S^{r e p}$ denote the set of SUs that perform sensing in the frame that are required to report their local decision to the CBS. Then, the total energy consumption for reporting is given by

$$
\mathrm{E}^{\mathrm{rep}}=\sum_{\mathrm{nEs} \text { rep }} E_{n}^{\text {rep }}
$$

The optimization for the spectrum sensing and the decision variable used for the optimization model is first defined:

Let $Y_{m n}=\left\{1\right.$, if channel $m$ is sensed by $\left.S U_{n}\right\}\{0$, otherwise $\}$

$$
W_{n}=\left\{1, \text { if } S U_{n} \text { transmit sensing result to } C B S\right\}\{0 \text {, otherwise }\}
$$

Note: $P_{m n}^{f}, P_{m n}^{d}$ denote the probability of false alarm and probability of detection, signal to noise ratio over channel $\mathrm{m}$ respectively.

It was assumed here that $P_{m, n}^{f}$ was fixed, then for a complex value phase shift keying (PSK) channel with circularly symmetric complex Gaussian noise $P_{m, n}^{d}$ became

$$
P_{m, n}^{d}=U\left(\frac{U^{-1}\left(P_{m n}^{f}\right)-\sqrt{T_{m n} F_{s} W_{m n}}}{\sqrt{2 W_{m, n}+1}}\right)
$$

$F_{S}=$ sample frequency, $\mathrm{U}=$ complementary cumulative distribution of standard Gaussian, $T_{m n}=$ time from equation 3.3, for a given $P_{m n}^{d}$ value, the required $T_{m n}$ can be written as

$$
T_{m n}=\left(\frac{U^{-1}\left(P_{m n}^{f}\right)-U^{-1}\left(P_{m n}^{d} \sqrt{2 W_{m . n}+1}\right)}{\sqrt{W_{m, n} F_{s}}}\right)^{2}
$$


In addition, let $T_{m, n}^{\min }$ denote the sensing time required for $\mathrm{S} U_{n}$ in order to achieve $P_{m, n}^{d}$ value of 0.5 . It can be calculated from equation 3.3

$$
T_{m, n}^{\min }=\frac{U^{-1}\left(P_{m, n}^{f}\right)}{Y_{m, n} \sqrt{F_{s}}}
$$

If assumed in this model that channel should be sensed by at least $\delta^{\min } S U S$. Then, $\delta^{\text {min }}$, defines the minimum number of co-operating $\mathrm{S} U_{S}$ for a channel. The selection of $\delta^{\mathrm{min}}$ is a design criterion. In order to encourage co-operation and improve robustness, a $\delta^{\text {min }}$ value greater than one is used in this design. Assuming that $P_{m, n}^{f}=P^{f} \forall_{m n}$ then $Q_{m}^{f}$ is given by

$$
Q_{m}^{f}=1-\prod_{n E S_{n}}\left(1-P^{f}\right)
$$

Since $Q_{m}^{f} \leq$ th $Q^{f}$, then the maximum number of co-operating SUs, denoted by $\delta^{\max }$, can be calculated as

$$
\delta^{\max }=\left[\frac{\log \left(1-\operatorname{th} Q^{f}\right)}{\log \left(1-P^{f}\right)}\right]
$$

In other words, $\delta^{\max }$ is the maximum number of co-operation SUs that satisfy the co-operation false alarm constraints. The solution methodology applied here can also be used for the case where $P_{m, n}^{f}$ value differs. The optimization problem can be written as

\section{- Optimization problem}

$$
\min _{w}=\sum_{m=1}^{m} \sum_{m=1}^{m} P^{s} T_{m, n}+\sum_{n=1}^{N} E_{n}^{r e p} Y_{n}
$$

Subject to

Where $Q_{m}^{d}$ is defined as

$$
\begin{gathered}
T_{m n} \geq T_{m n}^{\min } Y_{m, n} \forall_{m} \in M, \forall_{n} \in N \\
\sum_{m=1}^{N} T_{m, n} \leq T_{y_{n}}^{s} \forall_{n} \in N \\
\sum_{n=1}^{N} X_{m, n} \geq \delta^{m i n} \forall_{n} \in M \\
\sum_{n=1}^{N} X_{m, n} \leq \delta^{\max } \forall_{n} \in M \\
\sum_{m}^{m} X_{m, n} \leq m Y_{n} \forall_{n} \in N \\
\sum_{m=1} \\
t^{d}-m_{m}^{d} \leq O \forall_{n} \in M \\
X_{m, n} Y_{n} \in\{0,1\} \forall_{n} \in M, \forall \forall_{n} \in \\
T_{m n} \geq 0 \forall_{m} \in M, \forall_{n} \in N
\end{gathered}
$$

$$
Q_{m}^{d}=1-\prod_{n-1}^{N}\left(1-U\left(\frac{U^{-1}\left(P^{f}\right)-\sqrt{T_{m, n} F_{s} Y_{m n}}}{\sqrt{2 Y_{m n}}+1}\right) X_{m, n}\right)
$$

However, SUs with $X_{m, n}$ value of 0 and 1 to the above multiplication, whereas with $X_{m . n}$ value of 1 contribute $\left(1-P_{m, n}^{d}\right)$

The objective function in (3.8i), minimizes the energy consumption associated with sensing for frame. Constraint (3.8a) denotes the total time spent by an SU for sensing should be less than or equal to the sensing duration of frame. It also forces all $T_{m, n}$ value with SUn to zero if $Y_{0}=0$.

Constraint (3.8b) specified that if SUn senses channel $\mathrm{m}$, the sensing duration should be at least $T_{m, n}^{\mathrm{min}}$.

Constraint (3.8c) requires that each channel should be sensed by at least $\delta^{\text {max }}$ SUs.

Constraint (3.8d) limits the number of co-operating SUs for a channel in order to satisfy the false alarm probability threshold.

Constraint (3.8e) forces $Y_{n}$ value for an SU to 1, if that SU senses any channel. The requirement for cooperative detection probability being greater than the threshold for each channel is expressed by constraint $(3.8 \mathrm{~g})$, and finally, $(3.8 \mathrm{~h})$ and (3.8i) specify the type of variables 
In the algorithm $1, S^{r e p}$ is a set of SUs that are going to perform sensing and transmit their report for frames. Similarly, $S^{\text {nrep }}$ is the set of SUs that are not assigned to sense channel yet. Initially, $S^{r e p}=\Phi$, $S^{\text {nrep }}=\left\{S U_{1}, S U_{2}, \ldots S U_{N}\right\}$. The algorithm at first looks for SUs among $S^{r e p}$ in order to save reporting energy. If SUs are not found, then it moves on to $S^{n r e p}$. SUs in $S^{r e p}$ and $S^{n r e p}$ are processed in decreasing order of $Y_{m n}$ value for the considered channel. Each channel is sensed with $\delta^{\min }$ SUs. The required $P d$ value is calculated with:

This guarantees a minimum detection probability of $P_{\text {min }}^{d}$

$$
P d=\frac{\max \left(1-\left(1-t h Q^{d}\right)\right.}{\delta^{\min }} ; P_{\min }^{d}
$$

Where $T^{s}$ is the sensed direction for a frame.

$T^{s}$ is given by: ${ }_{n}^{\max }\left[\sum T_{m, n}\right]$

\subsection{Analysis and Evaluation}

The analysis at this juncture is for the execution of SU bandwidth requirement. The spectrum selection component requires this execution. The selection of this design explains how it estimates the bandwidth required by this SU. Hence the average path loss and Signal-to-noise ratio experienced by this SU was executed. Thus, using AMC, the bandwidth required for this SU is estimated.

\subsubsection{Data Collection and Analysis.}

The data used for this work was collected from Globacom mobile operator. This is based on the Huawei RFSS network controller. The P25 channel controller of the RFSS radio system manages radio access, assignment gather, and record network traffic statistics. For the data collecting snapshot of traffic radio network, statistics was collected from the traffic log of Huawei P25 RFSS radio network controller. From the network traffic snapshot, the parameters of RF environment, and the performance data was extracted and tabulated in Table 3.1. The radio network parameters such as numbers of channels, transmit power, burst (slot) duration are configurable for proper simulation. The data sampling window is 4 seconds, number of channel radio frequency channel is 14 , and the Channel bandwidth (channel capacity) is $10^{5} \mathrm{~Hz}$, transmission power of 15 watts and Bus (slots) duration, 625 seconds. The record of occupied and unoccupied channels, number of PUs and SUs, access spectrum sensing duration, wait queue and active radio sessions, and network parameters were used for the setup and configuration of the simulation environment. Occupied channel means when the PUs are active while unoccupied channel are holes that are underutilized by PUs which form the basis of SUs activities.

\subsubsection{Path Loss Evaluation.}

The algorithm program code uses the long distance path loss model to estimate the path loss in the maritime cognitive radio network. This model is used only in radio for distance beyond the far field distance from cognitive radio transmitter $d^{f}$, which depends on the maximum linear dimension of transmitter antenna (DA) and wavelength $\left(\lambda=\frac{C}{f}\right.$ where $C$ is the speed of light $)$ and can be estimated.

$$
P L(d)=P L(d o)\left[\frac{d}{d o}\right]^{n}
$$

Where $\mathrm{n}$, is the path loss component, (typically $2<n<6$ ); that indicates the rate at which the path loss increases with d also $P L(d)$ is the path loss at $d o$.

It is notable that in practice $d o$ is in the order of the cognitive radio transmitter antenna dimension for example, for cellular phone operating at $900 \mathrm{mHz}$ band with $\mathrm{DA}=5 \mathrm{c}, d^{f}$ is $33 \mathrm{c}$. Consequently, the probability that two cognitive radio users that are separated by distance lower than do is very small. That is, $\operatorname{Pr}(d<d o \approx 0)$.

Table 3.1. Network Traffic Statistic Collection [34]

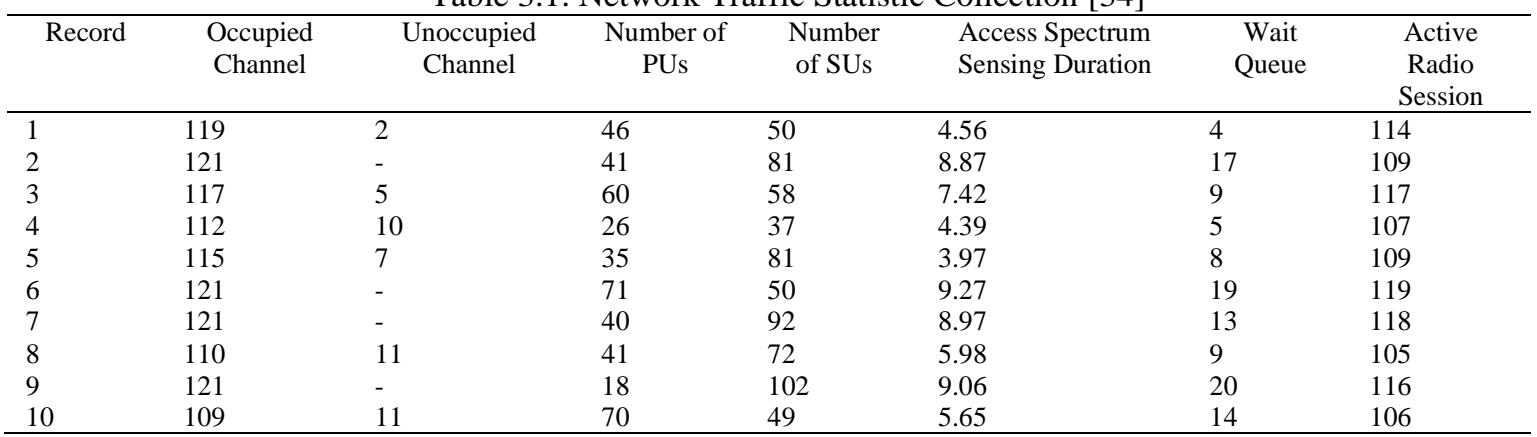




\section{SIMULATION RESULTS AND DISCUSSION}

The developed demand based spectrum allocation algorithm was implemented in the java programming language. The digital model of the case study cognitive radio network was created using the advanced Cisco packet tracer 7.0. The packet tracer is a network modeling simulation program for the design and simulation of infrastructure (wired or wireless). It has the facilities to emulate enterprise network device, protocol and algorithms. The Cisco packet tracer program has Application Programming Interface (API) support for java and $\mathrm{C}^{++}$programming language. With this program codes of algorithm (such as that of the developed demand based spectrum allocation algorithm) can be loaded into its workspace, enabling it to interact with the kernel of the packet tracer program. The demand based spectrum allocation algorithm program interacts with the packet tracer radio network operating system object via Common Object Request Broker Architecture (CORBA). This program allows inter object communication. It allows communication between object that are written in different programming language.

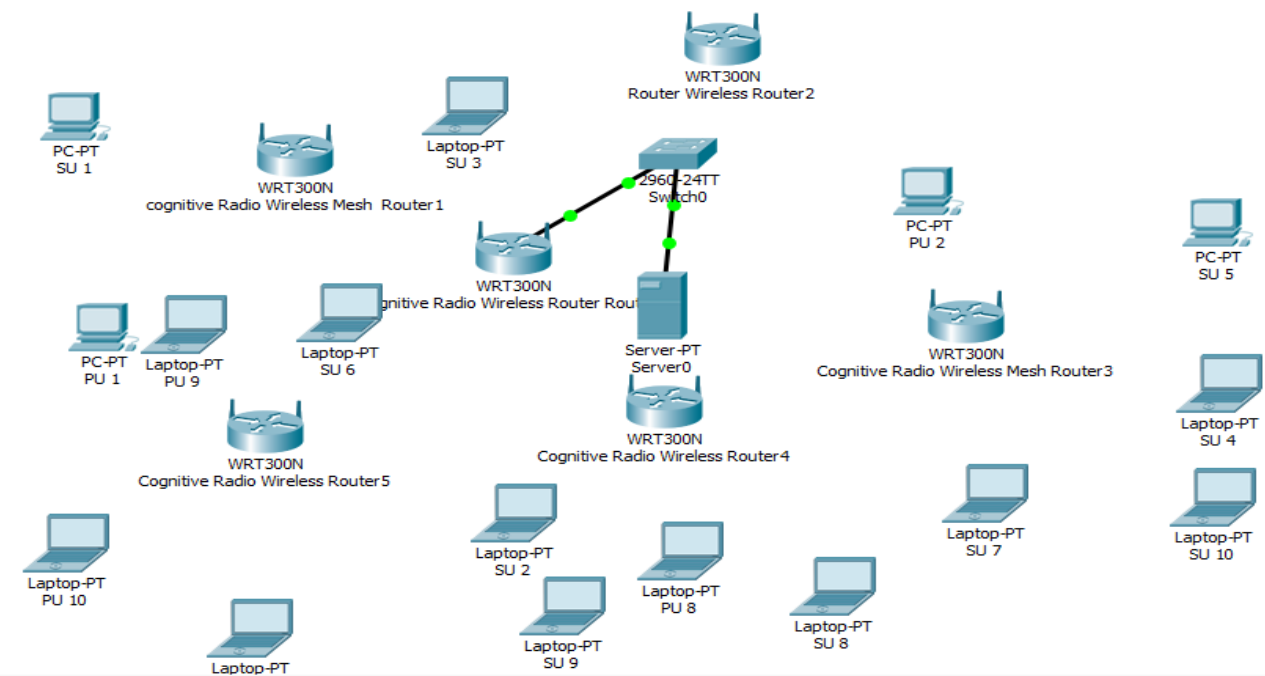

Figure 4.1 Maritime cognitive for evaluating the performance of developed demand based spectrum allocation algorithm

\subsection{Analysis of Results}

Figure 4.1 shows the case study cognitive radio maritime network created using the Cisco packet tracer software. The model consists of 5 mesh network routers and 20 mesh client (PUs and SUs). Of the 20 mesh client, 10 are PUs and the other 10 are SUs. The program allows configuration of the network router radios characteristics, the parameters of the layer 2 switches and the mesh client. In the packet tracer environment, the radio network parameters such as numbers of channels, transmit power, burst (slot) duration are configurable in other to properly setup the simulation. Data used for the setup and configuration of the simulation environment is shown in Table 3.1. In the packet tracer network model in Figure 4.1 cognitive maritime radio wireless router 0 is setup as mesh controller. It has a wired connection to the base server. As the mesh controller, it runs the spectrum allocation and de-allocation program in the network. Background program script interacts with the packet tracer radio network operating system.

The packet tracer's radio network operating system loads the mesh client access control script that runs the activations of the SUs and PUs based on Poisson distribution. This emulates the SUs requesting and yielding channels at random. The network management memory space (i.e. the Cisco packet tracer network operating system running configuration) of the mesh controller can be accessed programmatically to view network traffic statistic logs and trace files such as buffer size, transmit power, allocated/de-allocated channel numbers of client service session, the queue size and the routing table as the simulation runs. In the simulation, 60 iterations were done; and each iteration lasts for 60 seconds.

During each of the iteration, the network traffic statistics is queried programmatically from the simulation trace file in interval of three seconds (the network performance data sampling window is 4 seconds). From the simulation trace file, the network performance statistics are extracted and plotted to evaluate the performance of the developed algorithm. From the simulation trace file, the performance for throughput, spectrum counts, delays, bytes transferred, spectrum sensing duration, energy usage for sensing, processor time are extracted. The working of the genetic spectrum allocation algorithm is simulated using the same set of data in order to compare its performances with that of the developed algorithm. 


\subsection{Results Discussion}

\section{- Throughput Evaluation}

The evaluation and comparison of the variation of network throughput with time using the demand based algorithm and the genetic algorithm for spectrum allocation is shown in Figure 4.2. In the figure it can be seen that, for the demand based algorithm the throughput rises with time and almost stabilized to $154.6765 \mathrm{kbytes} / \mathrm{sec}$ at about $28.5 \mathrm{~seconds}$. The increased and steady throughput indicates effectiveness in the algorithm. This show that the PUs and SUs activities increase as holes' detection effort varies, that the algorithm kept the attained throughput at a good steady level. For genetic algorithm, the variation of throughput is shown (Figure 4.2), unlike in the case of the propose demand based algorithm, the throughput rose gradually. It got to its peak value of $113.5023 \mathrm{kbytes} / \mathrm{sec}$ at about 40.4657 seconds then it falls to 107.9117 Kbytes/sec at about 49.3246 seconds and gradually tappers to 103.7941 Kbytes at about 60 seconds. This indicates that the variation in throughput with the genetic algorithm is not stable as it is with the proposed demand based algorithm. This variation is as a result of algorithm not adapting properly to the increase in the opportunistic allocation and de-allocation of spectrums to secondary users. Hence its ability to co-ordinate dynamic spectrum sensing in the event of sustained random spectrum sensing by SUs result to less stable network throughput than with the case of the proposed demand based algorithm.

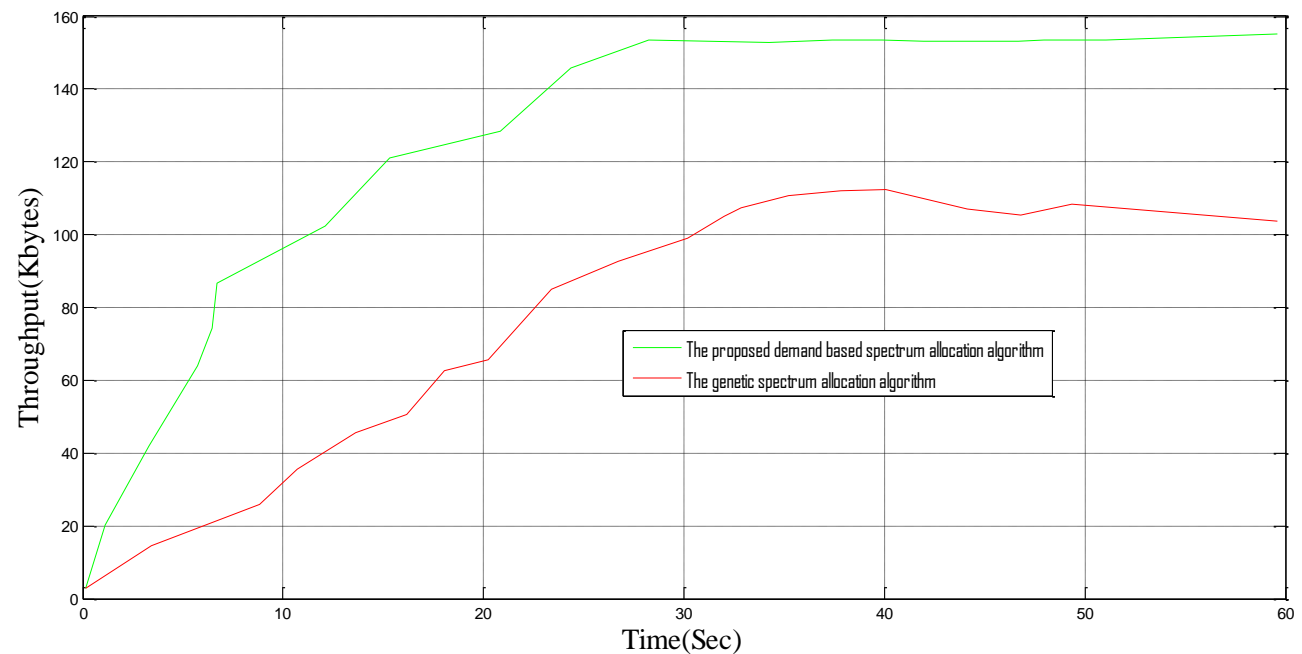

Figure 4.2 Comparison of the variation of network throughput using the demand based algorithm with the variation of throughput using genetic algorithm.

The simulation results show both models have high degree of correlation although the demand delay shows more degree of correlation with respect to sessions. Also, the generic based has more error of estimation due to high variance. This might result in collisions and possible retransmission of data or data loss. All these bring about energy wastages. The delay demand based has lower deviation and this is good..

From the simulation results it was shown that, the average throughput of the demand based algorithm is $117.0985 \mathrm{kbytes} / \mathrm{sec}$ while the average throughput of the genetic algorithm is $79.61495 \mathrm{kbytes} / \mathrm{sec}$. This shows that the proposed demand based algorithm outperforms the genetic algorithm by $44.15 \%$.

\section{- Delay Evaluation}

Figure 4.3 shows the delay with respect to communication session comparison for the genetic and the developed demand based algorithm. For the genetic algorithm plot, it can be seen that as the number of session established increases (SUs or PUs can establish more than one session), the delay increases. Non effective spectrum allocation especially the situation where spectrum holes exist but the allocation algorithm is not effectively computing and allocating holes to mesh client (PUs and SUs) that needs to communicate, this leads to increase in delay as seen with genetic algorithm. The demand based algorithm is far less delayed than in the genetic algorithm. The optimization in the spectrum allocation using demand based algorithm impact effectively in the reduction of the delay from that obtain using the genetic algorithm for allocating spectrum. The average delay in the radio network using the propose algorithm is $3.88 \mathrm{sec}$, while that of the 
genetic algorithm is $8.58 \mathrm{sec}$. From this result the developed algorithm achieved approximately $54.7 \%$ improvement in the delay reduction over the genetic algorithm.
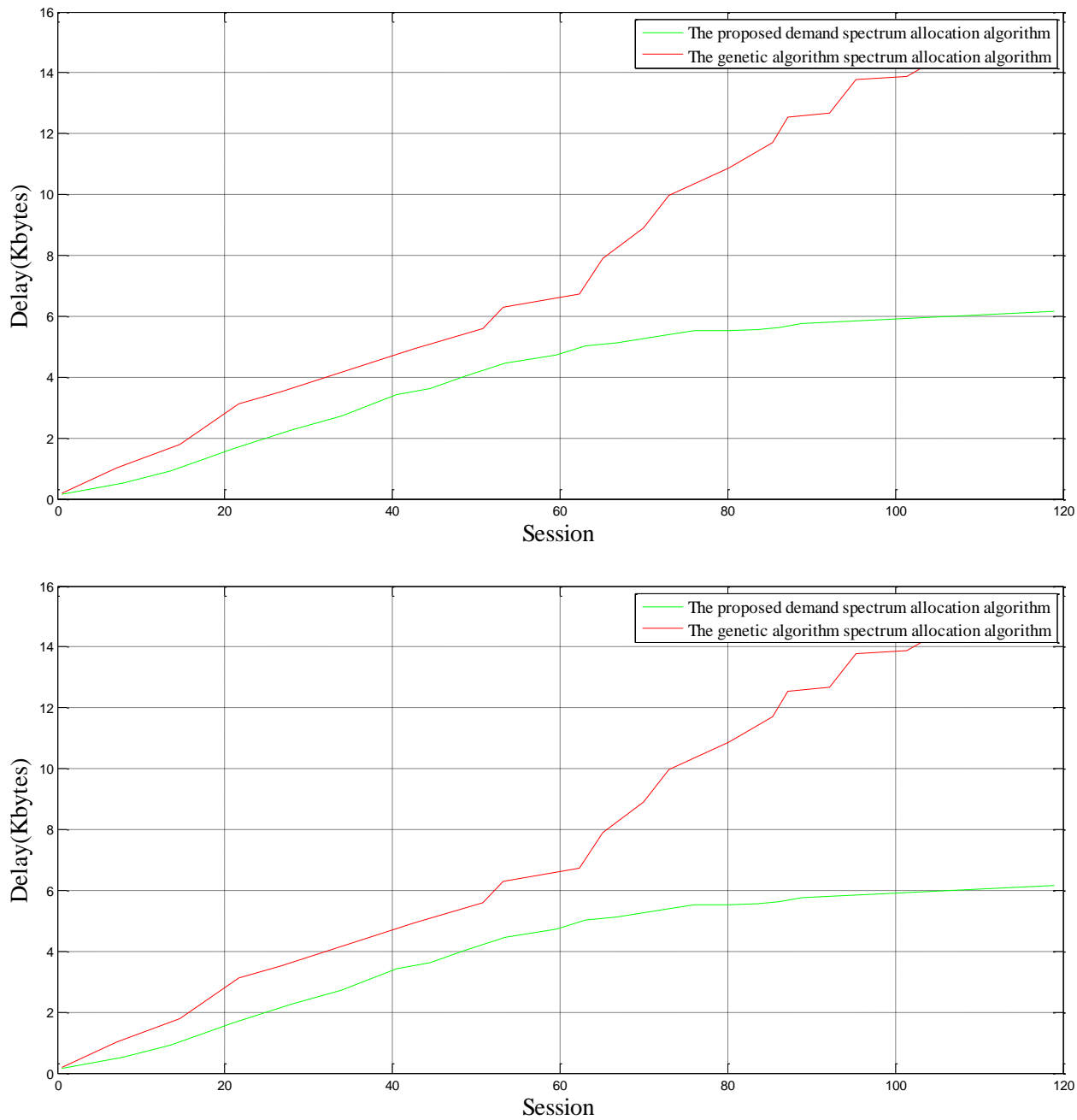

Figure 4.3 Comparison of the network delay using the demand based with the genetic algorithm

\section{- Maritime Utility Evaluation}

The variation of system utility (efficiency of spectrum usage with increase in SUs ratio of spectrum usage to SUs) comparison of the demand based and genetic spectrum allocation algorithm is shown in Figure 4.4. It can be seen that the utility reduces with increase in the number SUs that come online. For the genetic protocol, the utility falls steadily from about $100 \%$ (default) to about $25 \%$ in about 20 seconds. Hence, the developed demand based algorithm variation of utility with SUs is not as steady as that of the genetic algorithm. The demand based algorithm system utility falls from about $100 \%$ (the default value) to about $56 \%$ in seconds. The algorithm seems to maintain the utility at this value (it actually kept the utility slightly varying around this value). This indicates stability in the usage of radio spectrum. We also observed from figure 4.4, that the utility resulting from the pressure of SUs have greater impact for the genetic algorithm than it does for the demand based algorithm. The reduction in utility is at a greater rate for the genetic algorithm than for the propose demand based algorithm.

The average utility of the network using the propose demand based algorithm spectrum allocation algorithm is $72.02 \%$ and that of the genetic is $60.5 \%$. The demand based algorithm achieved a better spectrum utility (usage) than that of the genetic algorithm. These represent $12.52 \%$ improvement over the genetic algorithm in spectrum hole usage. 


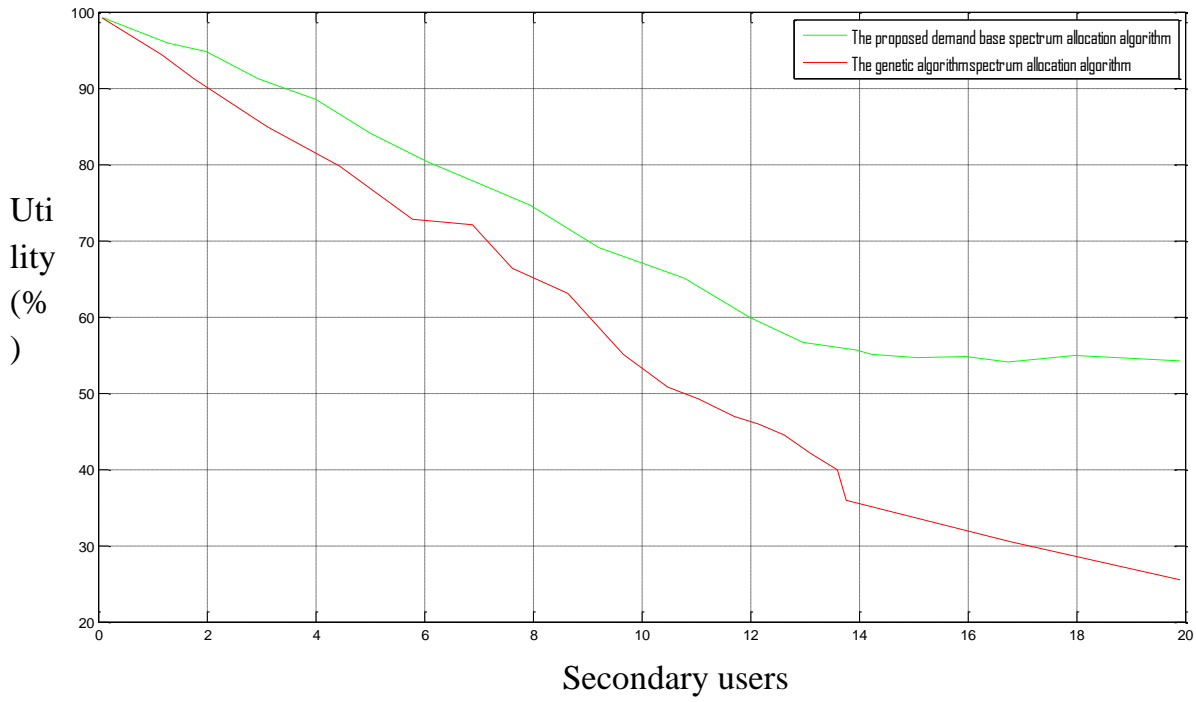

Figure 4.4 Comparison of utility of the proposed demand based and genetic algorithm

\section{- Sensing Power Evaluation}

Figure 4.5 shows the increase of sensing energy comparison of the genetic and demand based algorithm with increase in secondary user spectrum opportunistic access. As more secondary users gain radio access, the sensing energy increases. However, the amount by which this increase also depends on the versatility of spectrum allocation algorithm. The more versatile the frequency allocation algorithms the more efficient spectrum holes are discovered thus the less in the increase of sensing energy required. The increase in the sensing energy is not as steady as that of the genetic algorithm. The energy increase is from about 15 watts to about 35.88 watts with entrants of at least 20 SUs. One thing this does is that the detection of spectrum holes using the proposed algorithm is more efficient than using the genetic algorithm. This relate directly to the effectiveness and versatility of the spectrum allocation algorithm.

Figure 4.5 distinctly shows the difference in the sensing energy requirement for the two algorithms. The average sensing energy for the genetic algorithm and that of the demand based algorithm are 33.80watts and 25.13 watts respectively. Percentage of improvement is approximately $26 \%$. This shows appreciable level of optimization made possible through demand based algorithm.

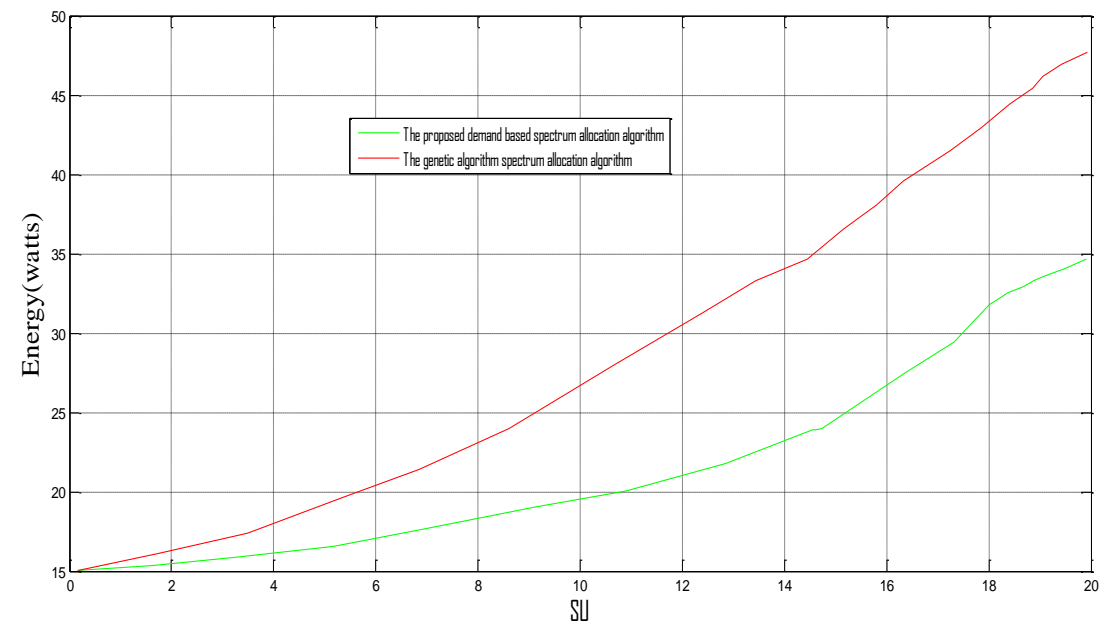

Figure 4.5 Variation of sensing energy with SUs for genetic and proposed demand based algorithm.

5.

\section{CONCLUSION}

The problem of dynamic spectrum allocation was tackled in this work using demand based algorithm. This algorithm was designed to optimally decide, based on the secondary user to assign available channel and spectrum sensing minimization. The minimization of spectrum sensing energy is the objective function of the optimization algorithm, while the optimization constraints include; limit on cooperating SU 
for channels, flags indicating whether SU sensed a channel or not and sensing time limit. The demand based algorithm was implemented in the java program language in order to test its performance. Advanced Cisco packet tracer (7.0 Software) was used to create a digital model of the cognitive radio environment.

Simulation results showed the effectiveness of the developed demand based algorithm. Comparative analysis indicates that the developed demand based algorithm outperformed the genetic algorithm. The variation of throughput with time indicates the developed demand based algorithm maintained the stability of throughput even with the increase in SU radio connection session. A higher average throughput was achieved in the developed algorithm than the genetic algorithm by a margin of $44.15 \%$. The use of demand based algorithm result has far less traffic delays than the genetic algorithm. The delay experienced by genetic algorithm was reduced by about $54.7 \%$.

There was an effective impact in the improvement of the utility of the radio network resource for the developed demand based algorithm. A higher rate of system resource utilization was achieved over the genetic algorithm. The average utility of the network resource using the demand based algorithm is $72.02 \%$ while that of the genetic algorithm is $60.5 \%$. This indicates that the demand based algorithm realized $12.52 \%$ improvement over the genetic algorithm in spectrum hole usage.

Finally, the demand based algorithm reduced the spectrum sensing energy usage of the genetic algorithm by 8.67 watts. The low spectrum sensing energy used by the demand based algorithm indicates that it is versatile and more efficient compared to the genetic algorithm. This work shows appreciable level of optimization. The percentage of energy optimization is approximately $26 \%$.

\section{REFERENCES}

[1] K. A. Yau, A. R. Syed, W. Hashim, J. Qadir, C. Wu, and N. Hassan, "Maritime Networking : Bringing Internet to the Sea," IEEE Access, vol. 7, pp. 48236-48255, 2019, doi: 10.1109/ACCESS.2019.2909921.

[2] A. M. Rateb, "Technical report Introduction to Cognitive Radio Systems," no. February, 2019, doi: 10.13140/RG.2.2.19607.83363.

[3] A. R. Roselainy, "Universiti Teknologi Malaysia, Malaysia," Teach. Learn. Math. Univ. Lev., vol. 16, no. May, pp. 195-198, 2005, doi: 10.1007/0-306-47231-7_19.

[4] S. Ahmad and T. Hult, "Decision Making Techniques For Cognitive Radios," 2008.

[5] R. Ahmed and Y. A. Ghous, "Detection Of Vacant Frequency Bands In Cognitive Radio,” No. May, 2010.

[6] S. Lin, S. Member, and K. Chen, "Improving Spectrum Efficiency via In-Network Computations in Cognitive Radio Sensor Networks," vol. 13, no. 3, pp. 1222-1234, 2014.

[7] M. Haddad, A. M. Hayar, S. Antipolis, and P. De Moulon, "Spectral efficiency of Cognitive Radio systems," pp. 4165-4169, 2007.

[8] S. Chatterjee, "Cognitive Radio And Dynamic Spectrum Access - A Study Cognitive Radio And Dynamic Spectrum Access - A Study," no. February, 2016, doi: 10.5121/ijngn.2014.6104.

[9] R. Biswas, "Basics of Cognitive Radio Networks: An Appraisal," no. May 2016, 2017.

[10] G. P. Joshi, S. Y. Nam, and S. W. Kim, Cognitive Radio Wireless Sensor Networks: Applications, Challenges and Research Trends. 2013.

[11] Akyildiz I. F. and Xudong Wang, "A Survey on Wireless Mesh Networks”, IEEE Communication Magazine, 43(a): S23-S30, 2005.

[12] Tandra R. and Sahai A. "Fundamental Limits On Detection in Low SNR Indoor Noise Uncertainty "in proc. "IEEE Int. Conf. Wireless networks, Communication and Mobile Computing, Vol. 1, Main, Hi, pp. 484 -469, 2005

[13] J. S. Pathmasuntharam, J. Jurianto, P. Kong, Y. Ge, M. Zhou, and R. Miura, "Maritime Ship-to-Ship / Shore Mesh Networks,” 2007, doi: 10.1109/ITST.2007.4295914.

[14] I. F. Akyildiz, W. Lee, M. C. Vuran, and S. Mohanty, "NeXt generation / dynamic spectrum access / cognitive radio wireless networks : A survey," vol. 50, pp. 2127-2159, 2006, doi: 10.1016/j.comnet.2006.05.001.

[15] Yuan Y. P. Bahl, Chandra R., Chou P. A., Ferrell J. I, Moscibroda T., Narkanka S., and Wu Y. "Cognitive Radio Network Over White Spaces" in proc. IEEE int. Symposium On New Frontiers In Dynamic Spectrum Access Networks, Dublin, Ireland, , pp. 416-427, 2007.

[16] Tang. H. "Some Physical Layer Issues of Wide-Band Cognitive Radio Systems" In Proc. IEEE Int. Symposium On New Frontier in Dynamic Spectrum Access Networks, baltimore, Maryland, USA pp. 151-159, 2005.

[17] Cabric D., Tkachenko A., and Brodersen R. "Spectrum Sensing Measurement of Pilot, Energy and Collaborative Detection" In Proc. IEEE Mitilant Communication. Washington, D. C. USA. pp. 1-7, 2006.

[18] Yuicek T. and Arslan H, "Spectrum Characterization for Opportunistic Cognitive Radio Systems" In proc. IEEE Military Communication Cong; Washington, D. C, USA, pp. 1- 6, 2006.

[19] Liang, Y. zang Y, E.peh, and Hoang. A; "Sensing Throughput Tradeoff for Cognitive Radio Network”, IEEE Transaction on wireless communication, volume .7, No 4, pp.13-26, 2008.

[20] Jayasri B. S., G. Raghavendra Rao. 'A novel predictive optimization scheme for energy-efficient reliable operation of a sensor in dynamic scenarios"; International Journal of Electrical and Computer Engineering. Vol. 10, No.1, February 2020, pp. 323 332. 


\section{BIOGRAPHIES OF AUTHORS}

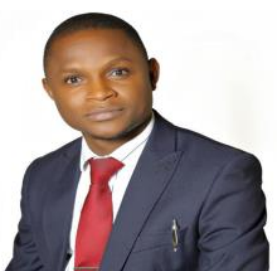

Onu, Dickson E. obtained his B.Eng. Electrical/ Electronic Engineering and MSc Maritime Telecommunication from University of Agriculture, Makurdi and University of Nigeria, Nsukka in 2011 and 2018 respectively. He is presently working with Multi-Power Engineering Limited as an Engineer and a Safety consultant at the International Breweries Sagamu, Nigeria. His research interest include superheterodyne radio receiver, and Dynamic Spectrum Allocatio Accessing Cognitive Radio Network in a maritime. Email: onudickson@gmail.com

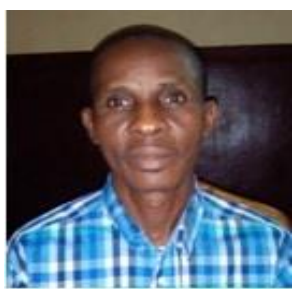

Ahaneku, Mamilus A. obtained his B.Eng. Electrical/Electronic Engineering and MSc Communications Engineering from Federal University of Technology, Owerri, Nigeria in 1994 and 2000, respectively. He holds $\mathrm{PhD}$ in Communications Engineering from University of Nigeria, Nsukka. He is a Senior Lecturer in the Department of Electronic Engineering, University of Nigeria, Nsukka. His research interests include Wireless Communication, Micrcowave Systems and Radio Frequency Design. E-mail: mamilus.ahaneku@unn.edu.ng or ahamac2004@yahoo.co.uk

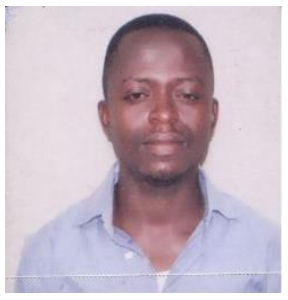

Ezea, Michael O. obtained his B.Eng Electronic Engineering and MSc. Marine Telecommunication from University of Nigeria Nsukka in 2013 and 2018 respectively. He is a lecturer II in the Department of Electrical Engineering, Nigeria Maritime University, Okerenkoko, Delta state, Nigeria. His research interest includes but not limited to wireless sensor networks, error detection techniques in wireless communication. Email: ezea.michael@unn.edu.ng or mikezeah@gmail.com

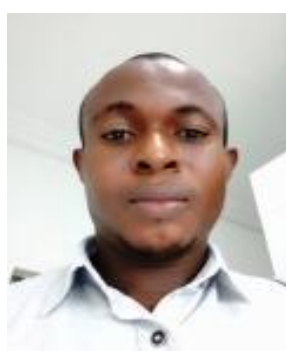

Osuagwu, Henry O. obtained his B.Eng. Electronic Engineering and M.Eng. Connunication Engineering from University of Nigeria, Nsukka, Nigeria in 2011 and 2016 respectively. He is currently a Ph.D. student in the Department of Electronic Engineering, University of Nigeria, Nsukka, Nigeria. He is a member of Nigerian Society of Engineers (NSE) and a registered COREN. He is a Lecturer in the Department of Electrical Enigeering, Nigeria Maritime University, Okerenkoko, Delta State, Nigeria. His research interests include Wireless Communication, Radio Resource Management, and Cognitive Radio in Wirelesss Sensor Networks. Email: henry.osuagwu.pg68120@unn.edu.ng or osuagwuhenry4justice@yahoo.com

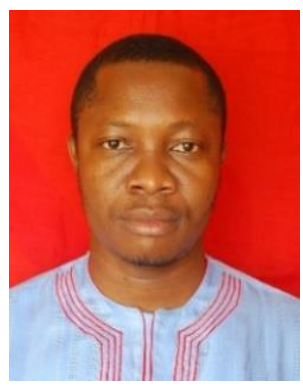

Nwawelu, Udora Nwabuoku received the B. Eng. M. Eng. and Ph.D degrees in Electronic Engineering from University of Nigeria, Nsukka in 2007, 2012, and 2019, respectively. His current research interests include wireless technology and networks, especially in the area of radio resource management and optimization. He has published several scholarly works in national and international journals E-mail: nwabuoku.nwawelu@unn.edu.ng 